\title{
A Laboratory on the Microprocessor Control of a Floating Ping Pong Ball
}

\author{
A. Dellah, P.M. Wild, B.W. Surgenor \\ Department of Mechanical Engineering \\ Queen's University, Kingston, Canada
}

\begin{abstract}
An undergraduate laboratory has been developed for a course in mechatronics that involves the control of a floating ping pong ball with a microprocessor. The apparatus consists of a ping pong ball located in a vertically oriented Plexiglas tube. At the top end of the tube, an ultrasonic transducer measures the position of the ball in the tube. A small cooling fan at the bottom end of the tube is used to control the height of the ball. Proportional-Integral-Derivative (PID) closed loop control is implemented by means of the microprocessor. In the laboratory, the students are required to program the microprocessor and conduct experiments in controller tuning. This paper describes a mechatronics laboratory that is easy to duplicate and exposes the students to various mechatronics issues.
\end{abstract}

\section{Introduction}

Since its introduction as an elective in 1997, the Mechatronic Systems Design (MECH 452) course offered through the Department of Mechanical Engineering at Queen's University has become very popular. This is reflected in positive student feedback and with a class size higher than the average for an elective course in Mechanical Engineering at Queen's (45 versus the average of 25). The course and its popularity are not isolated cases, but follow a growing trend towards including "mechatronics" type courses in traditional Mechanical Engineering curriculums ${ }^{1}$. However, the availability of resource material specific to "mechatronics" courses is still, like the courses, in an early stage of development.

Although there are widely differing interpretations of the meaning of the word "mechatronics", a common feature of courses entitled "Mechatronics" is the use of hardware oriented laboratories involving apparatus with dedicated microprocessor control. For example, faculty at San Jose State University have been running a "mechatronics engineering laboratory" since 1995 ". Their laboratory sequence has 12 experiments. Initially students are required to build and work on the analog control of simple electro-mechanical systems. Near the end of the sequence, students have progressed to more complex microprocessor controlled mechatronic systems. Thus, the experiments range from a light controlled switch assembled from basic electronic components to a microprocessor controlled parts sorter with multiple inputs and outputs. The latter involves a stepper motor driven conveyor belt paired with a table top electric robot in combination with photoemitters for part detection. Students are required to interface and program the microprocessor to sort parts between bins.

A slightly different approach was taken at Cal Poly where the facility for laboratory experiments is referred to as the "Mechatronics Design Studio" 3 . The experiments are more group project 
oriented. The most common project involves the design, construction and programming of a table top mobile (wheeled) robot. Another project is similar to San Jose's parts sorter in that students are required to program a robot to move small parts around. However, at Cal Poly the parts are delivered by one of the mobile robots from the other projects.

As a third example, Mariapan at GMI developed a sequence that he believes allows the students to acquire a complete mechatronics product design experience ${ }^{4}$. The experimental sequence is similar to San Jose's in that the students begin with simple analog temperature control and finish with microprocessor control of a mobile robot. Care is taken to emphasize the multi-disciplinary nature of mechatronics which is generally accepted as being fundamentally different from traditional mechanical laboratories. Each experiment is intended to have the following activities:

- Understanding the problem, identification of objectives and variables to be controlled

- Understanding the physical principles of the sensors and the process to be controlled

- Selection of the appropriate control algorithm and nature of the interface

- Wiring and calibrating the system

- Developing and implementing the computer program

It's fair to say that the above list of lab activities is common to the majority of mechatronic laboratories. What may differentiate these activities from conventional automatic controls laboratories is the much more hands-on nature (wire it up !) and system orientation (deal with the sensors and the D/A \& D/A interfacing) elements of the exercise.

At Queen's University a sequence of six laboratories is used:

1) Series and parallel resistive circuits, use of multimeters and oscilloscopes

2) Introduction to programming of the Basic Stamp microprocessor

3) D/A conversion, R/C time constants, analog control of a DC motor

4) Op-amp based analog signal acquisition and conditioning

5) Basic Stamp speed control of a DC motor with Darlington transistors

6) Basic Stamp PID control of the Ping-Pong apparatus

The labs are one part basic electric circuits and electronics, and one part introductory microprocessors, with motor control as the application. The contention is that students readily pick up on the details associated with sensors and actuators, but it is the microprocessor and the electronics of the interface that present the greatest difficulty. Although the ping pong apparatus is a more basic experiment than that involving say a mobile robot, it contains all the elements of a mechatronic systems (sensors, actuators, interfacing, microprocessor) and exposes the students to the underlying practical and theoretical issues.

\section{Description of the Apparatus}

The ping pong ball apparatus is illustrated in Figure 1. The apparatus consists of a cooling fan (taken from a PC) located at the base of a vertical tube (in which the ping pong ball levitates) and an ultrasonic sensor at the top of the tube (to measure the height of the ball). The tube is $3 \mathrm{ft}$ long with an OD of 2 in. The speed of the fan is controlled by the microprocessor using a form of digital Pulse-Width-Modulation (PWM). The ultrasonic sensor is an analog device and as 


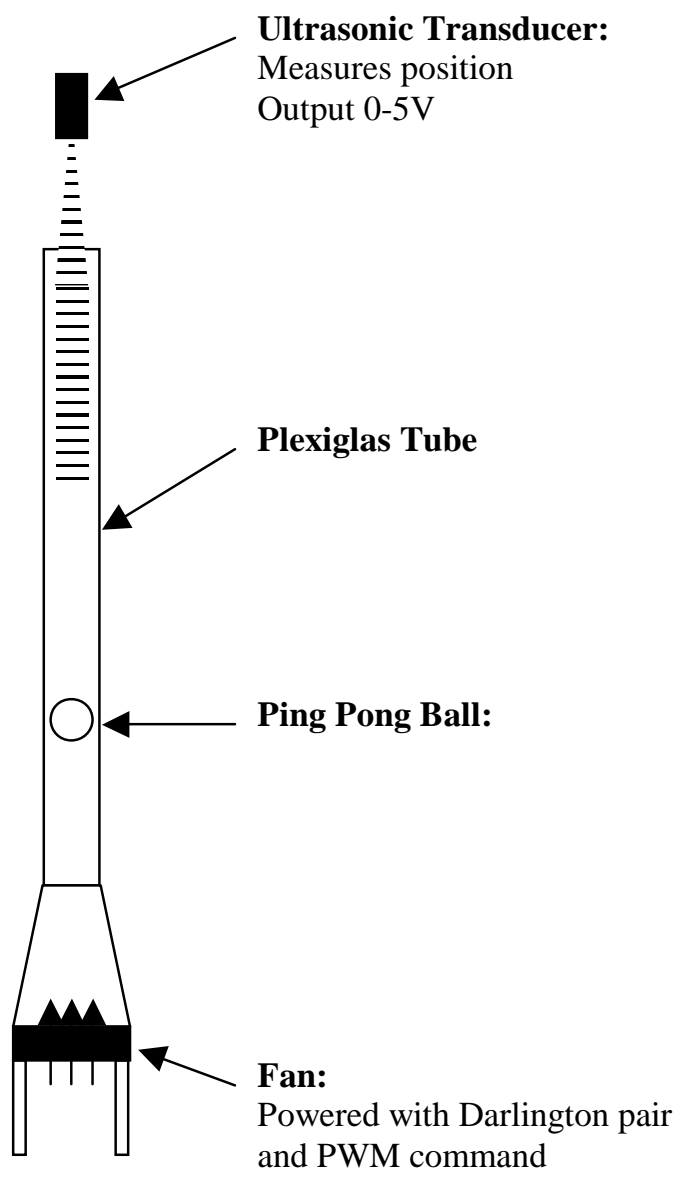

Figure 1. Illustration of the ping pong apparatus

such an A-to-D converter is used to feed the ball height back to the microprocessor and provide formal closed loop control. The control algorithm mimics a full three mode PID controller, with Proportional, Integral and Derivative action. The design of the apparatus is based upon that originally proposed by Cantrell ${ }^{5}$. The estimated cost of materials and components for each apparatus (including the microprocessor and the ultrasonic sensor) is approximately \$US150. Two pieces of apparatus have been constructed to date with plans to double the number.

The microprocessor is a Basic Stamp 2 that runs programs written in PBASIC. This is a programming language developed by Parallax Inc and is a hybrid of BASIC. The Stamp has sixteen fully programmable I/O pins that can be directly interfaced with TTL-level devices such as LEDs, manual switches, potentiometers and A/D converters. Programming is performed offline on a PC connected to the Stamp by a serial cable.

The Basic Stamp is a relatively inexpensive device with a wide range of applications reported. For example, the Stamp is used in an "Introduction to Engineering" course at the University of Minnesota with an enrollment of just under 200 students ${ }^{6}$, with individual students tasked with constructing a Stamp controlled mobile robot. Texts are also now available that give application details for the Stamp ${ }^{7}$. At Queen's, the Stamp was placed on a prototyping board that included a 
9 VAC power supply jack and short circuit protected I/O connections. Figure 2 shows the prototyping board with the Stamp microprocessor located to the left of the capacitor. Diagrams for the input and the output circuits are shown as Figure 3. At the point in the course where this lab occurs, the students are generally familiar with these circuits and components. For example, the motor driver output circuit shown in Figure 3(a) includes: a low pass RC filter to convert the pulse width modulated signal from the Stamp to a DC level; a buffer amplifier to minimize the load on the Stamp and; closed loop control of motor voltage with the op-amp and Darlington

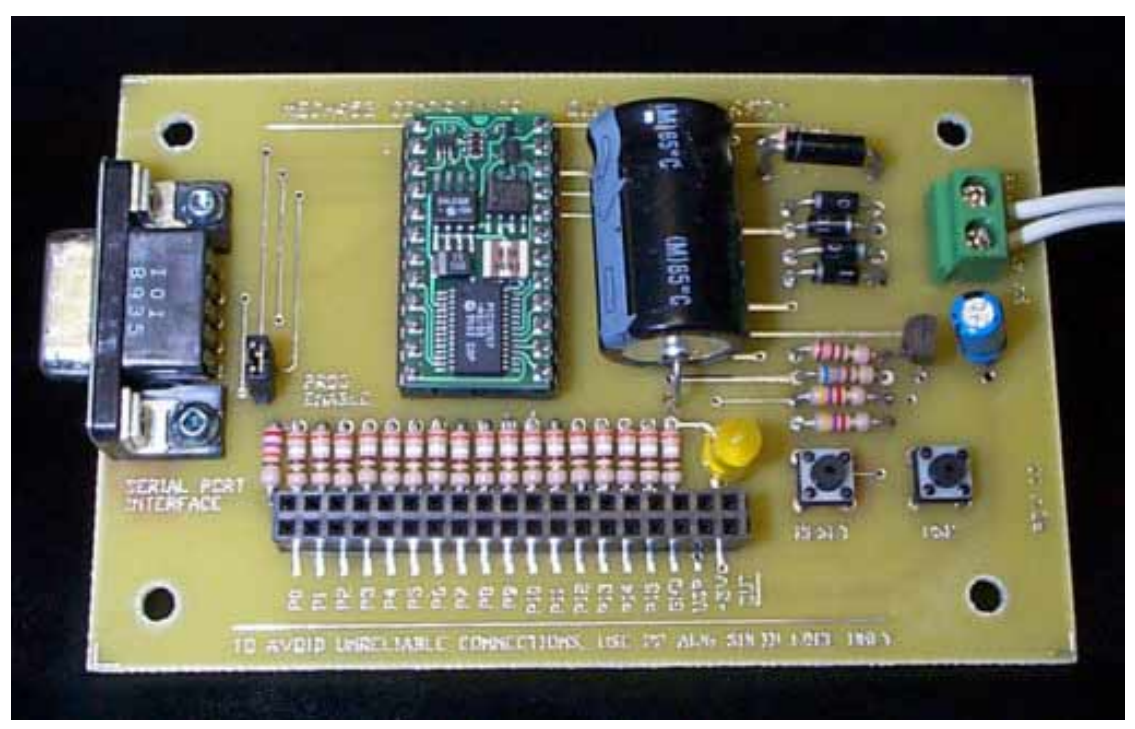

Figure 2. Basic Stamp microprocessor and prototyping board

(a)

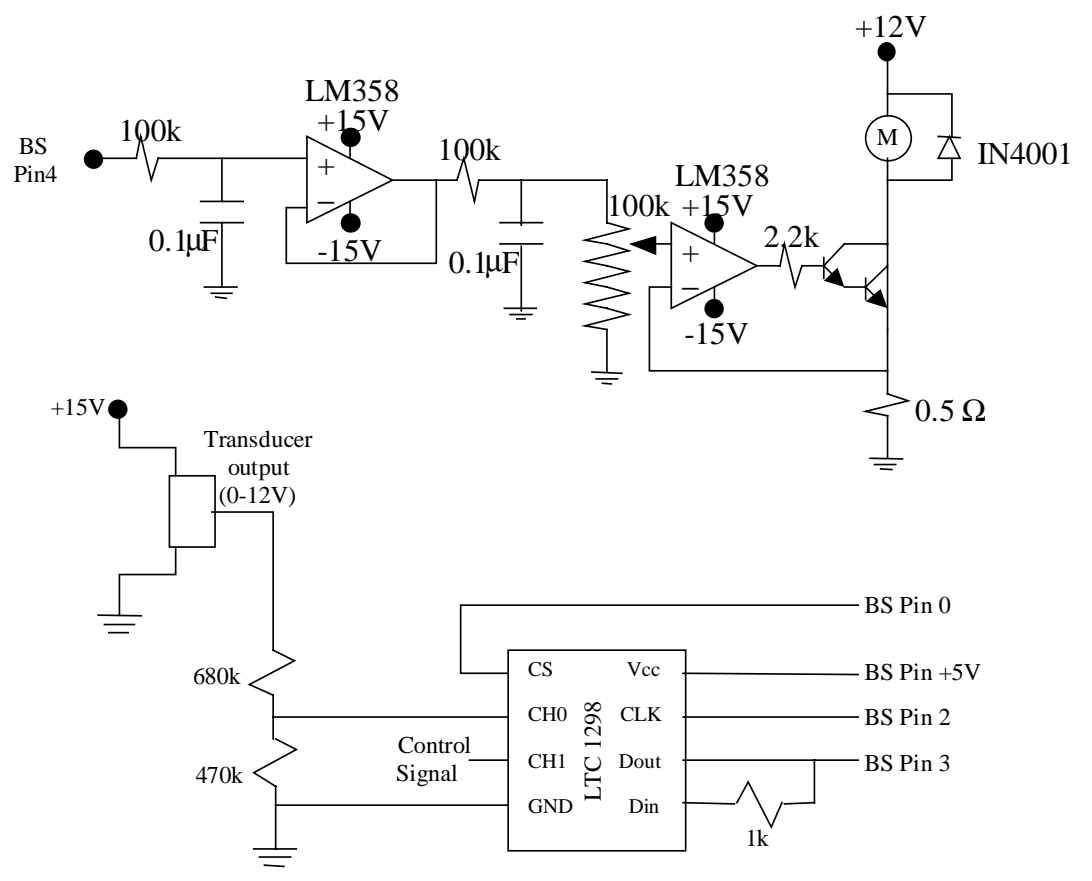

Figure 3. Circuit diagrams: (a) motor driver output and (b) sensor A/D input 
pair transistors. All of these circuits, as well as the connection of the LTC1298 A/D converter input circuit shown in Figure 3(b), are covered in previous labs and circuit design exercises.

\section{Description of the Laboratory}

In the laboratory, the students are required to: 1) assemble the apparatus, 2) program the microprocessor with a PID algorithm, and 3) tune the PID controller. Aside from getting the apparatus to function, the objective is to maximize the ability of the device to track five different kinds of height reference signals: straight (pure regulation), sine, triangle, sawtooth and square waves. A LabVIEW ${ }^{\mathrm{TM}}$ based data acquisition and control program has been developed to both generate the reference signal and to determine a performance score. This performance score is calculated using the measure illustrated in Figure 4.

Table 1 gives sample performance scores for the different height reference wave types. The students are provided with this table so that they can have a measure of the success of their own control algorithm. But, before the students can set out to maximize their performance, they have to deal with the limitations of the microprocessor as they relate to sampling time, lack of floating point and lack of negative numbers. Once they have dealt with these issues, they have to develop an empirical tuning procedure for the PID controller.

Figures 5 and 6 illustrate some sample tuning results. Three traces are shown: the reference signal (which alternates between a square wave and a triangular wave), the measured height and the control signal to the fan motor. These particular results were for a rule based control algorithm which selected a fan speed according to the size of the position error. Figure 5 shows a poor result which used a two speed (high or low but never off) algorithm. The low speed was sufficient to allow the ball to fall to the bottom of the tube. This result is seen to produce a highly oscillatory (yet marginally stable) response.

By adopting a rule based algorithm with 12 speeds the response is much improved and a sample result is shown as Figure 6. The 12 speed algorithm is a form of proportional gain scheduling controller and the 2 speed algorithm is a basic on-off controller. The introduction of a PID algorithm with PWM introduces a much more complicated tuning exercise for the students. One

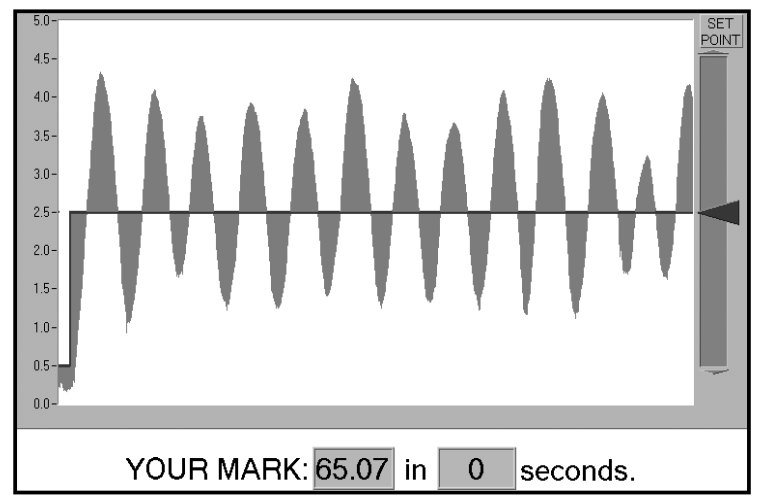

$$
\text { Performance Score }=\frac{\text { Total Area }-2 \times \text { Error }}{\text { Total Area }} \times 100
$$

Sample calculation \#1:

"Sample Program's response to a step input"

Error $=$ Grey Area $\quad=52.4$ Volt-Seconds

Total Area $=$ White Area + GreyArea $=300$ Volt-Seconds

$$
\text { Performance Score }=\frac{300-2 \times 52.4}{300} \times 100=65 \%
$$

Figure 4. Sample result with performance score calculation (60 sec time scale) 
Table 1. Sample performance scores for different height reference wave types

\begin{tabular}{|c|c|c|}
\hline Wave type & Performance Score & Highest Score to Date* \\
\hline \hline & $69.30 \%$ & $96.19 \%$ \\
\hline & $76.84 \%$ & $93.03 \%$ \\
\hline & $72.34 \%$ & $92.76 \%$ \\
\hline$\square$ & $60.53 \%$ & $88.85 \%$ \\
\hline Final Grade: & $61.46 \%$ & $83.42 \%$ \\
\hline
\end{tabular}

Effort needed to do any worse.

notes that there is a higher level of noise in Figure 6 relative to Figure 5, a problem that students could deal with through appropriate application of an analog filter.

\section{Conclusions}

The apparatus was found to be an invaluable supplement to the theory taught in the classroom, and in particular in the areas of microprocessor programming and interfacing, areas that are traditionally foreign to Mechanical Engineering students. After completing the laboratory, the students will have been exposed to the following aspects of a mechatronics system:

- Digital versus analog signals

- PWM control of an electric motor

- Off line programming of a microprocessor

- Programming limitations of a microprocessor

- Digital implementation of a PID control algorithm

- Procedures associated with tuning of a control algorithm

Feedback from students in the 1998 offering of this course was very positive. This is reflected in the fact that the enrolment for 1999 is up by close to $50 \%$ from 1998 . The popularity of mechatronics among the undergraduates bodes well for the planned development of a grouping of elective courses for students wishing to specialize in mechatronics. The current Mechatronics System Design course will be an important part of this grouping.

\section{Acknowledgements}

The test results are simulations based upon the experimental results given in an Undergraduate Laboratory Report written by M. Leggat, N. Fredrickson, J. MacDonald and A. Watchorn. 


\section{References}

1. Wild, P.M. and Surgenor, B.W., "An Innovative Mechatronics Course for a Traditional Mechanical Engineering Curriculum”, 1999 ASEE Annual Conference Proceedings, Session 2566, Charlotte, NC, June 20 to 23, 1999.

2. Wang, J.C, Furman, B.J., Hsu, T.R., Hsu, P., Reischl, P. and Barez, F., "Mechatronics Engineering Laboratory Development at San Jose State University", 1997 ASEE Annual Conference Proceedings, Session 3666, 1997.

3. Mariappan, J., "A Laboratory for Mechatronics Courses", 1997 ASEE Annual Conference Proceedings, Session 3666, 1997.

4. Alptekin, S.E., "Development of a Mechatronics Design Studio", 1997 ASEE Annual Conference Proceedings, Session 3666, 1997.

5. Cantrell, T., "PID-Pong Challenge", Circuit Cellar INK: The Computer Applications Journal, Issue 42, January 1994.

6. Durfee, W.K., "A Hands-On Introduction to Engineering Course for Large Numbers of Students”, 1999 ASEE Annual Conference Proceedings, Session 3266, Charlotte, NC, June 20 to 23, 1999.

7. Edwards, S., "Programming and Customizing the Basic Stamp Computer", McGraw Hill, 1998.

\section{AARON DELLAH}

Aaron Dellah received his M.Sc. in Mechanical Engineering from Queen's University in 1998. Prior to that, he received his B.Sc. in Mechanical Engineering from the University of Waterloo in 1996. His research interests currently lie in the field of biomechanical engineering. He worked on the development of the ping pong apparatus while employed as a Research Engineer in Mechanical Engineering at Queen's University in Kingston.

\section{PETER WILD}

Peter Wild is an Assistant Professor in the Department of Mechanical Engineering at Queen's University in Kingston Ontario. He received his B.A.Sc. from the University of British Columbia in 1983 and spent the following seven years as a practicing design engineer. He received his Ph.D from the University of Victoria in 1994 and assumed his current position at Queen's University in 1996. Peter teaches machine design and mechatronics and conducts research in a variety of fields with strong collaborative links to industry.

\section{BRIAN SURGENOR}

Brian Surgenor is a Professor and the Head of the Department of Mechanical Engineering at Queen's University in Kingston Ontario. He received his B.Sc. from Queen's University 1977, his M.Sc. from McMaster University in 1979 and his Ph.D from Queen's University in 1983. He joined the faculty in the Department of Mechanical Engineering at Queen's University as a Lecturer in 1979. Brian teaches control theory and mechatronics and conducts research on model-based nonlinear control algorithms to pneumatic systems 

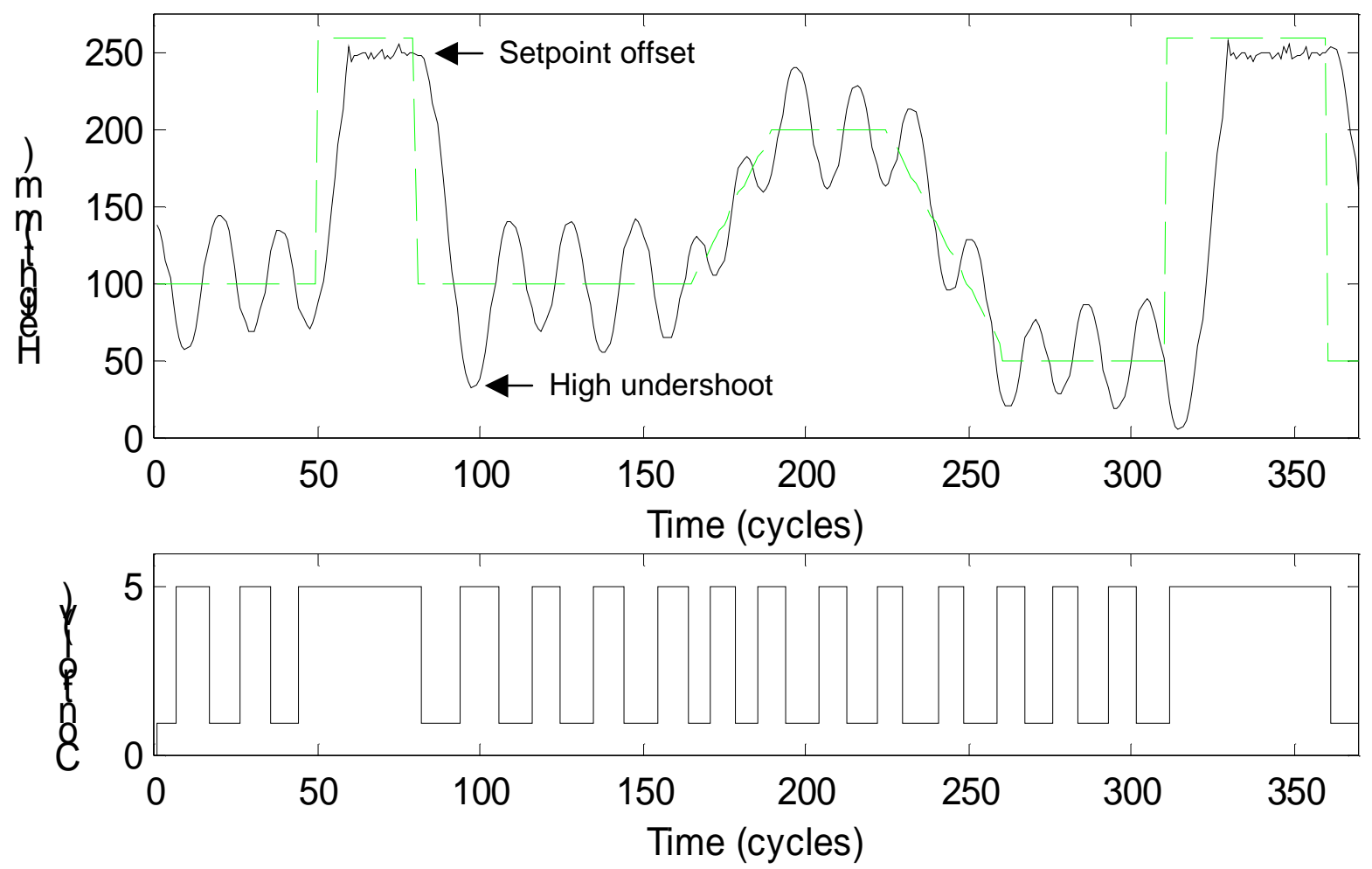

Figure 5. Sample response with poor control
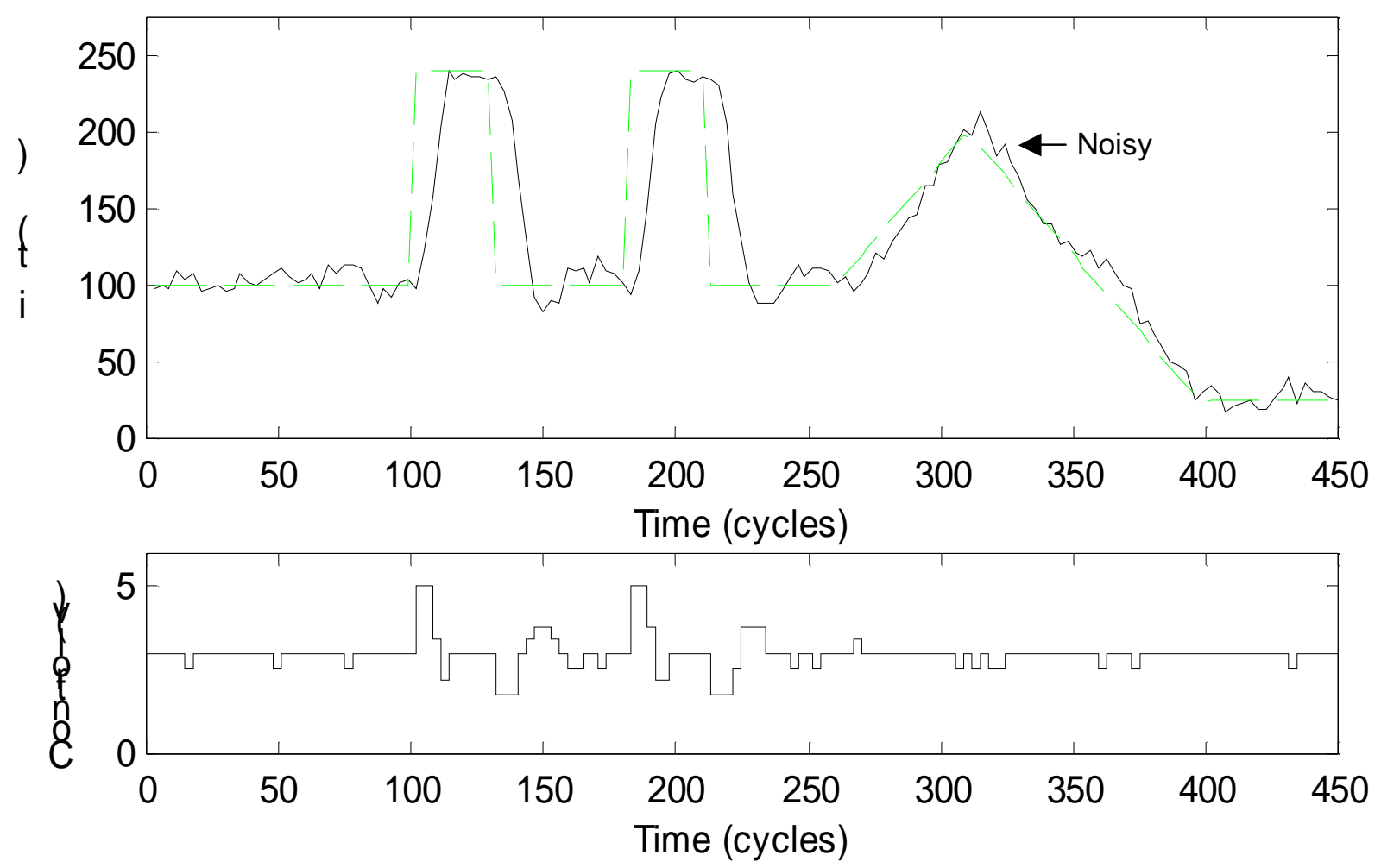

Figure 6. Sample response with better control 